\title{
A GENERALIZATION OF THE RIESZ-HERGLOTZ THEOREM ON REPRESENTING MEASURES
}

\author{
PETER A. LOEB ${ }^{1}$
}

\begin{abstract}
A simple construction is given that obtains maximal representing measures for positive harmonic functions on a domain $W$ as the weak* limits of finite sums of point masses on $[0,+\infty]^{W}$. This new standard result, new even for the unit disk, is established for very general elliptic differential equations and domains, in fact, for a Brelot harmonic space, using nonstandard analysis.
\end{abstract}

Let $\mathbf{C}$ denote the complex plane, $D$ the unit disk $\{z \in \mathbf{C}:|z|<1\}$, and $P(z, a)$ the Poisson kernel $\left(|z|^{2}-|a|^{2}\right) /|z-a|^{2}$. For each positive $r \leqslant 1$, let $C_{r}=\{z \in \mathbf{C}:|z|=r\}$ and let $\lambda_{r}$ be $1 / 2 \pi r$ times Lebesgue measure on $C_{r}$. Let $\Phi_{x_{0}}^{D}$ denote the positive harmonic functions on $D$ with value 1 at the origin $x_{0}$. By a theorem first established by F. Riesz [11] but usually attributed to G. Herglotz [3], there is for each $h \in \Phi_{x_{0}}^{D}$ a unique representing measure $\nu_{h}$ on $C_{1}$; that is,

$$
h(a)=\int_{C_{1}} P(z, a) d v_{h}(z)
$$

for each $a \in D$. Moreover, $\nu_{h}$ is the weak* limit as $r \rightarrow 1$ of the measures $h \lambda_{r}$ on $C_{r}$. Note that $\nu_{h}$ is both a measure on $C_{1}$ and on the harmonic functions $\left\{P(z, \cdot): z \in C_{1}\right\}$; these functions are the extreme points in the convex set $\Phi_{x_{0}}^{D}$

For a general open and connected set $W$ in a Euclidean space or Riemann surface, we let $\Phi_{x_{0}}^{W}$ denote the positive harmonic functions on $W$ with value 1 at some $x_{0} \in W$. Now it is the Martin boundary theory ([9] or [2]) and in general the Choquet theory [10] (both of which, historically, have roots in the Riesz-Herglotz Theorem) that give for each $h \in \Phi_{x_{0}}^{W}$ a unique representing measure $\nu_{h}$ (maximal with respect to the Choquet ordering) on the extreme elements of $\Phi_{x_{0}}^{W}$. The general Choquet theory does not give a simple construction of $\nu_{h}$. Martin's theory does allow a repetition for $W$ of the construction of $\nu_{h}$ for the disk, but one must imbed $W$ in its Martin compactification $W^{M}$. For many simple domains such as the Lebesgue spine,

Received by the editors July $18,1977$.

AMS (MOS) subject classifications (1970). Primary 31A10, 31B10, 31D05; Secondary 02H20.

Key words and phrases. Herglotz Theorem, representing measures, Brelot harmonic spaces, nonstandard analysis, weak* convergence.

'This work was supported by a grant from the U. S. National Science Foundation. 
the structure of $\bar{W}^{M}$ and, therefore, of $C\left(\bar{W}^{M}\right)$ is still unclear. It was only in 1970, for example, that a deep and difficult paper by Hunt and Wheeden [4] established the equivalence between Martin's boundary and the Euclidean boundary for a Lipschitz domain.

In this note, we give a simple construction of representing measures that is new even for the unit disk and is valid for very general elliptic differential equations and domains. For the disk $D$ and $h \in \Phi_{x_{0}}^{D}$, this construction is obtained by modifying the classical construction as follows:

For each positive $r<1$ and each $x \in D$ with $|x|<r$, let $\mu_{x}^{r}$ denote harmonic measure on $C_{r}$ with respect to $x$. That is, $\mu_{x}^{r}=P(\cdot, x) \lambda_{r}$ and for the origin $x_{0}, \mu_{x_{0}}^{r}=\lambda_{r}$. Let $\mathscr{\rho}_{r}$ be a partition of $C_{r}$ into a finite number of intervals $A_{i}$ with points $y_{i}$ chosen from each $A_{i}$, and let $\delta_{y_{i}}^{r}$ be unit mass at $y_{i}$ for each $i$. A trivial modification of the classical construction of $\nu_{h}$ as the weak ${ }^{*}$ limit of the measures $h \lambda_{r}$ shows that the measures

$$
\sum_{i} h\left(y_{i}\right) \mu_{x_{0}}^{r}\left(A_{i}\right) \delta_{y_{i}}^{r}
$$

converge to $\nu_{h}$ in the weak* topology as $r \rightarrow 1$ and the intervals in $\mathscr{P}_{r}$ become smaller. For our new construction, we replace each measure $\delta_{y_{i}}^{r}$ with unit mass $\delta_{i}^{r}$ on the harmonic function $\mu_{x}^{r}\left(A_{i}\right) / \mu_{x_{0}}^{r}\left(A_{i}\right)$ extended by 0 on and outside $C_{r}$. The measures

$$
\sum_{i} h\left(y_{i}\right) \mu_{x_{0}}^{r}\left(A_{i}\right) \delta_{i}^{r}
$$

converge to $\nu_{h}$ in the weak* topology for regular Borel measures on $[0,+\infty]^{D}$ as $r \rightarrow 1$ and the intervals in $\mathscr{P}_{r}$ become smaller. Note that by the StoneWeierstrass Theorem, the set of continuous real-valued functions on $[0$, $+\infty]^{D}$ is the uniform closure of the algebra generated by constants and all finite truncations of evaluations at points of $D$.

Our result is stated for a Brelot harmonic space $(\mathcal{H}, W)$ with 1 superharmonic on $W$. (See [2] or [5].) We do not need to add to Brelot's axioms the additional assumptions necessary for the Martin compactification theory. The reader who is interested in only a special case of the Brelot theory, e.g., solutions of Laplace's equation or more general elliptic differential equations in $R^{n}$, can read the rest of this note with the assumption that the case of interest is the one being discussed.

Fix $x_{0} \in W$. For each region (i.e., connected open set) $\Omega \subset W$, let $\mathcal{F}_{\Omega}=$ $\{h \in \mathcal{H}:$ domain $h=\Omega\}$. If $x_{0} \in \Omega$, let $\Phi_{x_{0}}^{\Omega}=\left\{h \in \mathcal{H}_{\Omega}: h>0\right.$ and $h\left(x_{0}\right)=$ 1). We assume that $\Phi_{x_{0}}^{W} \neq \varnothing$. Let $Y=[0,+\infty]^{W}$ with the product topology. The restriction of the product topology to $\Phi_{x_{0}}^{W}$ is the topology of uniform convergence on compact sets, and $\Phi_{x_{0}}^{W}$ is a compact metric space in this topology. (See [6] and [8].) Let $E_{x_{0}}$ be the set of extreme points in the compact convex set $\Phi_{x_{0}}^{W} ; E_{x_{0}}$ is a $G_{\delta}$ in $\Phi_{x_{0}}^{W}$ and therefore is a Borel set in $Y$. By Choquet's Theorem, for each $h \in \Phi_{x_{0}}^{W}$, there is a unique regular Borel measure $v_{h}$ supported by $E_{x_{0}}$ such that for each $x \in W$ 


$$
h(x)=\int_{E_{x_{0}}} T_{x}(g) d v_{h}(g) .
$$

Here $T_{x}$ denotes evaluation at $x$. (See [6].)

Given a region $\Omega$ in $W$, we let $\bar{\Omega}$ denote its closure and $C(\partial \Omega)$, the continuous real-valued functions on $\partial \Omega=\bar{\Omega}-\Omega$. We call $\Omega$ a regular inner region if $\bar{\Omega}$ is compact and each $f \in C(\partial \Omega)$ has a unique continuous extension $h_{f}$ on $\bar{\Omega}$ such that $h_{f} \mid \Omega \in \mathcal{H}_{\Omega}$ and $h_{f} \geqslant 0$ if $f \geqslant 0$. Since the mapping $f \rightarrow h_{f}$ is positive and linear, for each $x \in \Omega$ there is a Radon measure $\mu_{x}^{\Omega}$, called harmonic measure for $x$ and $\Omega$, on $\partial \Omega$ such that for each $f \in C(\partial \Omega)$,

$$
h_{f}(x)=\int_{\partial \Omega} f d \mu_{x}^{\Omega} .
$$

If $h \in \mathcal{F}_{\Omega}, h \geqslant 0$, we extend $h$ with the value 0 on $W-\Omega$ so that $h \in Y=$ $[0,+\infty]^{W}$.

Let 9 consist of sets of the form $\alpha=\{\varepsilon, K\}$, where $\varepsilon$ is a positive real number, and $K$ is a compact set in $W$ with $x_{0} \in K$. Given $\alpha=\left\{\varepsilon_{\alpha}, K_{\alpha}\right\}$ and $\beta=\left\{\varepsilon_{\beta}, K_{\beta}\right\}$ in 9 , we say that $\alpha \leqslant \beta$ if $\varepsilon_{\alpha} \geqslant \varepsilon_{\beta}$, and $K_{\alpha} \subset K_{\beta}$. Clearly, $(G, \leqslant)$ is a directed set.

Now fix $h \in \Phi_{x_{0}}^{W}$. Given $\alpha$ in $\mathcal{G}$, associate with $\alpha$ and $h$ a measure $\nu_{h}^{\alpha}$ as follows:

Choose a regular inner region $\Omega \supset K_{\alpha}$ [5, Theorem 4.3]. By the equicontinuity of $\Phi_{x_{0}}^{W}$ on $\partial \Omega$ [8], we may partition $\partial \Omega$ into a finite number of measurable sets $A_{i}, 1 \leqslant i \leqslant m$, so that for each function $f \in \Phi_{x_{0}}^{W}$,

$$
\sup _{A_{i}} f-\inf _{A_{i}} f<\varepsilon_{\alpha} \text {. }
$$

By discarding null sets, we may assume that $\mu_{x_{0}}^{\Omega}\left(A_{i}\right)>0$ for each $i$. Choose $y_{i} \in A_{i}, 1 \leqslant i \leqslant m$. Let $\delta_{i}^{\Omega}$ be the unit mass on the harmonic function

$$
\frac{\mu_{x}^{\Omega}\left(A_{i}\right)}{\mu_{x_{0}}^{\Omega}\left(A_{i}\right)} \in \Phi_{x_{0}}^{\Omega}, \quad x \in \Omega .
$$

Then $\delta_{i}^{\Omega}$ is a regular Borel measure on $Y$. Let $\nu_{h}^{\alpha}$ be the sum

$$
\sum_{i=1}^{m} h\left(y_{i}\right) \mu_{x_{0}}^{\Omega}\left(A_{i}\right) \delta_{i}^{\Omega}
$$

THEOREM. The regular Borel measures $\nu_{h}^{\alpha}$ converge with respect to the ordering $\leqslant$ on 9 to the representing measure $\nu_{h}$ supported by $E_{x_{0}}$ in the weak* topology for regular Borel measures on $Y$. That is, for each continuous realvalued function $F$ on $Y$,

$$
\lim _{\alpha} \int_{Y} F(y) d v_{h}^{\alpha}(y)=\int_{E_{x_{0}}} F(y) d \nu_{h}(y)
$$

Proof. The proof is nonstandard. It is a modification of the proof of Theorem 4.6 of [6]. Here, however, one uses arbitrary continuous functions $F$ instead of just point evaluations $T_{x}$. One may associate the domain $\Omega$ and 
partition $\left\{A_{i}\right\}$ in the proof of Theorem 4.6 of [6] with an arbitrary infinite $\beta$ in ${ }^{*} g$; that is $\beta>^{*} \alpha$ for each standard $\alpha \in 9$. Since for each infinite $\beta \in * g$

$$
\int_{* Y}^{*} F(y) d \nu_{h}^{\beta}(y) \simeq \int_{E_{x_{0}}} F(y) d \nu_{h}(y)
$$

the theorem follows from A. Robinson's criterion for convergence of nets [12, Theorems 4.2.4 and 4.2.5].

Note. One can use the existence of a countable exhaustion of $W$ by compact sets $K_{n}$ (see, for example, [6, p. 161]) to obtain a cofinal sequence $\nu_{h}^{\left\{K_{n}, 1 / n\right\}}$ in the net $\nu_{h}^{\alpha}$.

For results similar to the above, see [6, Theorems 4.8 and 4.9]. For other results using this nonstandard method of dealing with standard weak* convergence arguments in potential theory, the reader is referred to [6, Theorems 3.1 and 6.5]. Weak* convergence for general spaces is discussed by Anderson and Rashid in [1]; weak* cluster points and further examples are discussed by the author in [7].

\section{REFERENCES}

1. Robert M. Anderson and Salim Rashid, A nonstandard characterization of weak convergence, Proc. Amer. Math. Soc. 69 (1978), 327-333.

2. M. Brelot, On topologies and boundaries in potential theory, Springer-Verlag, Berlin, 1971.

3. G. Herglotz, Uber Potenzreihen mit positivem, reellem Teil imEinheitskreis, Ber. Verhandl. Sächs Akad. Wiss. Leipzig Math.-Phys. Klasse 63 (1911), 501-511.

4. R. Hunt and R. Wheeden, Positive harmonic functions on Lipschitz domains, Trans. Amer. Math. Soc. 147 (1970), 507-526.

5. Peter A. Loeb, An axiomatic treatment of pairs of elliptic differential equations, Ann. Inst. Fourier (Grenoble) 16 (1966), 167-208.

6. Applications of nonstandard analysis to ideal boundaries in potential theory, Israel J. Math. 25 (1976), 154-187.

7. Weak limits of measures and the standard part map (preprint).

8. Peter A. Loeb and Bertram Walsh, The equivalence of Harnack's principle and Harnack's inequality in the axiomatic system of Brelot, Ann. Inst. Fourier (Grenoble) 15 (1965), 597-600.

9. R. S. Martin, Minimal positive harmonic functions, Trans. Amer. Math. Soc. 49 (1941), 137-172.

10. R. Phelps, Lectures on Choquet's Theorem, Van Nostrand, Princeton, N. J., 1966.

11. F. Riesz, Sur certaines systèmes singuliers d'équations intégrales, Ann. Sci. Ecole Norm. Sup 28 (1911), 33-62.

12. A. Robinson, Nonstandard Analysis, North-Holland, Amsterdam, 1966.

Department of Mathematics, University of IllinoIs, Urbana, Illinois 61801 